\title{
Critical assessment of wet-chemical oxidation synthesis of silicon quantum dots $\uparrow$
}

\author{
Jonathan L. Wilbrink, ${ }^{\text {abc }}$ Chia-Ching Huang, ${ }^{\mathrm{b}}$ Katerina Dohnalova (iD) bc \\ and Jos M. J. Paulusse (D)*ac
}

Received 1st October 2019, Accepted 1st November 2019

DOI: $10.1039 / c 9 f d 00099 b$

Wet-chemical synthetic procedures are powerful strategies to afford fluorescent silicon quantum dots (Si QDs) in a versatile and scalable manner. However, development of $\mathrm{Si}$ QDs is still hampered by a lack of control over photoluminescence emission, in addition to synthesis and characterization complexities. The wet-chemical Si QD synthesis by oxidation of magnesium silicide $\left(\mathrm{Mg}_{2} \mathrm{Si}\right)$ with bromine $\left(\mathrm{Br}_{2}\right)$ was revisited and a control reaction was carried out where the silicon source was omitted. Both reaction conditions resulted in substantial quantities of fluorescent material. Moreover, a comparative analysis of their optical properties (UV-vis/fluorescence) revealed no apparent differences. Other characterization techniques also confirmed the resemblance of the two materials as ${ }^{1} \mathrm{H}$ NMR, FTIR and XPS spectra were nearly identical for both samples. Elemental analysis revealed the presence of only $2 \mathrm{wt} \%$ silicon in the Si QD sample. No evidence was found for the formation of significant amounts of Si QDs via this wet-chemical procedure.

\section{Introduction}

Quantum dots (QDs), also coined semiconductor nanocrystals, are nanoparticles that possess unique properties on the quantum level that differ from bulk properties. ${ }^{1,2}$ Depending on the particle size and shape, QDs display photoluminescence, following the quantum confinement model. ${ }^{1}$ As a consequence, by varying QD dimensions, emission can be tuned over a broad spectral range. Owing to their superior optical properties, such as size-dependent fluorescence, ${ }^{3-5}$ color definition ${ }^{6}$ and resistance against photobleaching, ${ }^{7,8}$ QDs are promising materials for a wide variety of applications, including photovoltaics, ${ }^{9-11}$ lighting

\footnotetext{
${ }^{a}$ Department of Biomolecular Nanotechnology, MESA+ Institute for Nanotechnology, TechMed Institute for Health and Biomedical Technologies, Faculty of Science and Technology, University of Twente, P.O. Box 217, Enschede, 7500 AE, The Netherlands.E-mail: J.M.J.Paulusse@utwente.nl

${ }^{b}$ Institute of Physics, University of Amsterdam, Science Park 904, 1098 XH Amsterdam, The Netherlands 'SpectriS-dot b.v., Wilsonstraat 34, 2131 PT Hoofddorp, The Netherlands

$\dagger$ Electronic supplementary information (ESI) available. See DOI: 10.1039/c9fd00099b
} 
and displays, ${ }^{6,12}$ and biomedical imaging. ${ }^{13-19}$ This application potential is, however, severely limited by the fact that conventional QDs typically contain toxic and/or scarce elements (e.g. Cd, In, Pb, Se or Te). Silicon quantum dots (Si QDs) signify an appealing, non-toxic and highly sustainable alternative..$^{\mathbf{2 0}-22}$

Synthesis strategies for Si QDs are typically divided into top-down and bottomup approaches (and combinations). ${ }^{16}$ In top-down approaches, Si QDs are prepared from larger macroscopic materials, e.g. silicon wafers, to construct nanoparticles, which can be done mechanically, ${ }^{23}$ as well as by etching with strong acids, such as HF. ${ }^{24}$ In addition, the group of Veinot and coworkers developed an effective route based on the thermal decomposition of hydrogenterminated silsesquioxane to produce Si QDs within a silica matrix, followed by liberation of the Si QDs by HF etching. ${ }^{4,25,26}$

In bottom-up approaches, Si QDs are prepared from molecular precursors. This includes syntheses by physical means, such as condensation of silane gas into Si QDs via laser pyrolysis ${ }^{27,28}$ or plasma synthesis. ${ }^{3,29,30}$ Other strategies that have been reported to yield Si QDs include decomposition of precursors in supercritical fluids ${ }^{31}$ and by microwave irradiation. ${ }^{32-34}$ Bottom-up approaches by chemical means are typically wet-chemical syntheses, where reactions are carried out in solution-phase. ${ }^{16,21,35,36}$ Strategies include reduction of $\mathrm{SiCl}_{4}$ (or $\mathrm{SiBr}_{4}$, and sometimes co-reaction with alkyltrichlorosilanes) using reducing agents, such as alkali metals (e.g. $\mathrm{Na}, \mathrm{K}),{ }^{37-39}$ alkaline earth metals $($ e.g. $\mathrm{Mg}),{ }^{40}$ sodium naphthalide ${ }^{41,42}$ or $\mathrm{LiAlH}_{4},{ }^{43-49}$ and in molten salt syntheses using active $\mathrm{Al}$ species. ${ }^{50,51}$ Synthetic routes towards Si QDs have also been based on the oxidation of silicon species, typically Zintl salts $\mathrm{XSi}_{n}$, where $\mathrm{X}$ is an alkali metal or alkaline earth metal). Common methods are the oxidation of these Zintl salts by $\mathrm{Br}_{2},{ }^{52-56}$ $\mathrm{NH}_{4} \mathrm{Br},{ }^{34,57-59}$ or $\mathrm{NH}_{4} \mathrm{Cl} .{ }^{60,61}$ Furthermore, Zintl salts have been reacted with $\mathrm{SiCl}_{4}$ to yield Si QDs in a metathesis type synthesis. ${ }^{62-64}$ Regarding synthesis strategies, several reviews are recommended for a more complete and detailed overview. ${ }^{16,21,36,65}$

Wet-chemical synthesis is generally more versatile, less laborious and highly scalable, while elevated temperatures can be avoided, although limited control over fluorescence emission remains a major challenge. ${ }^{16}$ Moreover, the number of reliable wet-chemical synthetic strategies towards Si QDs is still limited, with complications concerning synthesis and characterization hampering research into Si QDs, as well as their commercialization.

Fluorescence emission generally correlates well with Si QD size, ${ }^{66}$ although discrepancies between Si QD size and emission have been reported.$^{25}$ In particular for wet-chemical syntheses, primarily blue or green fluorescence emission is observed, regardless of the reported size. There is still debate as to whether quantum confinement is in fact the origin of photoluminescence in Si QDs. ${ }^{67-69}$ Bulk silicon is an indirect bandgap material, ${ }^{70}$ and even on the nanoscale it is often implicated that fluorescence emission is either inefficient or mostly governed by surface defects rather than quantum confinement effects. ${ }^{9,71}$ Moreover, such defects are frequently reported,,$^{9,69,71,72}$ e.g. due to nitrogen-containing species on $\mathrm{Si}$ QD surfaces $^{73}$ or particle oxidation. ${ }^{72,74}$ Interestingly, even two seemingly comparable Si QD samples, albeit synthesized via different procedures, displayed very different optical properties, i.e. blue and red emission. ${ }^{75}$ These differences were explained by the occurrence of nitrogen-containing impurities in the blue-emitting Si QDs. Emission of Si QDs was demonstrated by Wiggers and 
coworkers to change from red to blue upon exposure to air, confirming that blue fluorescence can be induced by oxidation. ${ }^{74}$ Surface-related fluorescence is typically undesirable, as the emission is no longer tunable by adjusting the particle size, and such particles are more susceptible to photobleaching. ${ }^{7}$ Although photoluminescence is frequently assigned to surface defects when the quantum confinement model cannot be applied, ${ }^{71,75}$ this is not necessarily true in certain cases, as discussed below.

Interestingly, side reactions occurring during Si QD synthesis may also induce the (majority of) fluorescence and obscure results. Recently, Oliinyk et al. criticized $^{76}$ the preparation of Si QDs via a one-pot microwave synthesis employing 3aminopropyltrimethoxysilane (APTMS) as the silicon source, as reported earlier by He and coworkers. ${ }^{32}$ Their criticism focused on discrepancies concerning the XRD data when comparing with other Si QD literature, ${ }^{77}$ the absence of XPS data, TEM images showing crystalline material that is inconsistent with silicon, and most strikingly, very comparable optical properties for materials prepared in the presence, as well as in the absence of the silicon source.

Purification of fluorescent nanomaterials has also been pointed out to be critical. $^{78-80}$ For instance, fluorescent by-products (e.g. small molecules) were frequently insufficiently removed..$^{78-82}$ Crystalline nanoparticles of molybdenum sulfide, as observed by TEM, were reported to display strong fluorescence emission, ${ }^{83}$ although more recently, smaller impurities were demonstrated to be the actual source of fluorescence. ${ }^{84}$ Hence, connecting the optical properties to a nanomaterial remains a considerable challenge, as also pointed out earlier by Nandi and coworkers, ${ }^{80}$ who particularly stressed the importance of careful interpretation of TEM data. ${ }^{79,85}$

Our investigations into the preparation of Si QDs via the oxidation of $\mathrm{Mg}_{2} \mathrm{Si}$ with $\mathrm{Br}_{2}$, a well-established, facile reaction generally resulting in high product yields, ${ }^{52,53}$ revealed that attempts to alter the optical properties by changing the reaction parameters, such as temperature, reaction time, concentration and reagents, remained unsuccessful. A lack of size-control (and thus control over the emission) was noted earlier, but remained unexplained. ${ }^{52}$ This prompted us to investigate this reaction in more detail. An in-depth comparative study on these Si QDs is presented here, and a comparison is made with material resulting from a silicon-free synthesis, where the Zintl salt was omitted. Both materials were characterized using a number of different techniques, including ${ }^{1} \mathrm{H}-\mathrm{NMR}$ spectroscopy, FTIR, TEM, elemental analysis, XPS, fluorescence spectroscopy, UV-vis spectroscopy, and SEC. Both products possessed highly comparable properties, hinting towards a mechanism that presumably does not (exclusively) produce $\mathrm{Si}$ QDs, but primarily fluorescent by-products.

\section{Experimental}

\section{Materials and methods}

Syntheses were carried out under a nitrogen atmosphere, unless stated otherwise. Glassware was dried before use. Solvents were dried over 4 A molecular sieves for at least $24 \mathrm{~h}$ before use.

$n$-Butyllithium ( $n$-BuLi, $2.5 \mathrm{M}$, in hexanes), chloroform-d (99.8 atom\% D) and $n$-octane $(\geq 97 \%)$ were obtained from Acros, bromine $\left(\mathrm{Br}_{2}, \geq 99.99 \%\right.$ trace metals basis), 9,10-diphenylanthracene, hydrochloric acid ( $\mathrm{HCl}, 37 \%)$, magnesium 
silicide $\left(\mathrm{Mg}_{2} \mathrm{Si}, \geq 99 \%\right.$ trace metals basis, -20 mesh), molecular sieves $(0.3 \mathrm{~nm}$, rods), sodium sulfate $\left(\mathrm{Na}_{2} \mathrm{SO}_{4}\right.$, anhydrous), and sodium thiosulfate $\left(\mathrm{Na}_{2} \mathrm{~S}_{2} \mathrm{O}_{3}\right.$, $\geq 98 \%$, anhydrous) were obtained from Sigma Aldrich, chloroform, ethanol, ethyl acetate and methanol were obtained from VWR, TEM grids were obtained from EM Resolutions (Sheffield, United Kingdom), and XPS substrates from SSENS (Hengelo, The Netherlands).

\section{Characterization}

Nuclear magnetic resonance (NMR) spectroscopy was performed on a Bruker 400 $\mathrm{MHz}$ or Bruker $600 \mathrm{MHz}$ spectrometer. Samples were measured in chloroform-d. Attenuated total reflectance (ATR) Fourier-transform infrared (FTIR) spectra were obtained by drop-casting samples from chloroform solution on a Bruker Alpha-p. Elemental analysis for carbon was performed on a Vario Micro Cube CHNSAnalyser from Elementar, for bromine on an 883 Plus IC from Metrohm, and for silicon on a Specord 50 Plus UV/vis spectrophotometer from Analytik Jena. Transmission electron microscopy (TEM) was performed on a Philips CM300STFEG transmission electron microscope operated at $300 \mathrm{kV}$. Samples were dropcast from a dilute chloroform solution on holey carbon films on a 200 mesh copper substrate. X-ray photoelectron spectroscopy (XPS) was measured on a PHI Quantera SXM with a monochromatic Al K $\alpha$ source (1486.6 eV). The carbon C 1s signal at $284.8 \mathrm{eV}$ was taken as the reference binding energy. Samples were dropcast from a chloroform solution on gold-coated glass substrates (20 nm gold). UVvis spectra were recorded on a Shimadzu UV-2401PC UV-vis spectrophotometer. Absorbance was kept below 0.05 for quantum yield determinations to avoid inner filter effects. Ethyl acetate was employed as the solvent for UV-vis and fluorescence spectroscopy. Fluorescence was measured on a Varian Cary Eclipse fluorescence spectrophotometer. Size exclusion chromatography (SEC) measurements were performed on a Waters Alliance e2696, equipped with a 2475 fluorescence detector, and a 2998 photodiode array detector to determine absorbance. Samples were run on an Agilent PLgel $5 \mu$ m MIXED-D column, using HPLC-grade chloroform as the eluent. Before measurement, samples were filtered on $0.2 \mu \mathrm{m}$ Whatman Spartan syringe filters.

\section{Synthesis of Si QDs}

Si QDs were synthesized, with only minor changes, according to earlier literature procedures. $^{52,53}$ Degassed $n$-octane $(100 \mathrm{~mL}, 0.615 \mathrm{~mol})$ was added to $\mathrm{Mg}_{2} \mathrm{Si}$ $(100 \mathrm{mg}, 1.30 \mathrm{mmol})$, followed by the addition of $\mathrm{Br}_{2}(0.54 \mathrm{~mL}, 10.5 \mathrm{mmol})$. Subsequently, the dispersion was stirred for $2 \mathrm{~h}$, whereupon the color changed from deep orange to pale orange. The dispersion was heated to reflux for $18 \mathrm{~h}$ (or $60 \mathrm{~h}$, isolated yield $37.8 \mathrm{mg}$ ). Then, the solvent was removed under reduced pressure, under an inert atmosphere. Fresh, degassed $n$-octane $(100 \mathrm{~mL}, 0.615$ mol) was added and the mixture was cooled on ice. $n$-Butyllithium in hexane (2.5 M, $2.09 \mathrm{~mL}, 5.22 \mathrm{mmol}$ ) was slowly added and run overnight for complete reaction, while the dispersion was allowed to warm up to room temperature. Unreacted $n$-butyllithium was quenched with excess methanol $(10 \mathrm{~mL})$, and left to react for $1 \mathrm{~h}$. Thereafter, the dispersion was filtered and extracted against aqueous $\mathrm{HCl}(1 \mathrm{M}, 100 \mathrm{~mL}, 1 \times)$ and distilled water $(100 \mathrm{~mL}, 3 \times)$. During extraction, the organic phase was deep orange, with some insoluble materials, especially at the 
solvent-solvent interface, which were discarded. The organic phase was dried over $\mathrm{Na}_{2} \mathrm{SO}_{4}$ and filtered, and the solvent was removed under reduced pressure. The obtained product was a dark brown material $(24.4 \mathrm{mg})$.

\section{Control synthesis}

The control synthesis was performed similarly to the procedure described above, with the only change being that the silicon source, $\mathrm{Mg}_{2} \mathrm{Si}$, was omitted during the synthesis. Initially, more coloration was observed during the reaction, due to the absence of the dark purple $\mathrm{Mg}_{2} \mathrm{Si}$. The obtained product was a dark brown material (24.5 mg).

\section{Reaction between $\boldsymbol{n}$-octane and $\mathrm{Br}_{2}$}

$\mathrm{Br}_{2}(0.54 \mathrm{~mL}, 10.5 \mathrm{mmol})$ was added to degassed $n$-octane $(100 \mathrm{~mL}, 0.615 \mathrm{~mol})$ and reacted for $2 \mathrm{~h}$ at room temperature. Upon reaction, the orange color of the $\mathrm{Br}_{2}$ slowly fades. Subsequently, samples were drawn and analyzed with ${ }^{1} \mathrm{H}-\mathrm{NMR}$ spectroscopy.

\section{Results and discussion}

\section{Synthesis of Si QDs by oxidation of $\mathrm{Mg}_{2} \mathrm{Si}$ by $\mathrm{Br}_{2}$}

To investigate the structural and optical properties of the nanomaterials prepared via the oxidation of $\mathrm{Mg}_{2} \mathrm{Si}$ by $\mathrm{Br}_{2},{ }^{52-56}$ as depicted in Scheme 1, this synthesis was carried out with only minor changes: most importantly, commercial $\mathrm{Mg}_{2} \mathrm{Si}$ was used instead of that synthesized from $\mathrm{Mg}$ and $\mathrm{Si}$, solvent was removed at reflux temperature, rather than at room temperature, and surface passivation with $n$ butyllithium was carried out for 1 day, instead of for 2 days. The products of these reactions here are denoted as Si QDs (in the presence of $\mathrm{Mg}_{2} \mathrm{Si}$ ) and control (in the absence of $\mathrm{Mg}_{2} \mathrm{Si}$ ).

The products were first characterized using ${ }^{1} \mathrm{H}$ NMR spectroscopy, as shown in Fig. 1. The spectrum for Si QDs is in accordance with spectra published before. Signals at $0.88 \mathrm{ppm}$ and $1.25 \mathrm{ppm}$ were observed and have been assigned previously to $-\mathrm{CH}_{3}$ and $-\mathrm{CH}_{2}$ - protons of the butyl surface passivation, respectively. ${ }^{86}$ Similar peak broadening was observed as previously reported..$^{53}$ However, no proof for silicon-bound $-\mathrm{CH}_{2}-$ protons around $0.6 \mathrm{ppm}$ (ref. 87) was found, although whether such signals can be observed for these kinds of material is still the subject of debate. ${ }^{88}$ In comparison with the control, no obvious differences between the spectra are apparent. Signal intensity was low for both samples, even at high concentrations $\left(>20 \mathrm{mg} \mathrm{mL}^{-1}\right)$, while no precipitation was observed.

(a) $\mathrm{Mg}_{2} \mathrm{Si}+\mathrm{Br}_{2} \frac{\mathrm{n} \text {-Octane }}{\begin{array}{l}\text { 1.2 } \mathrm{h} \mathrm{RT} \\ 2.18 \mathrm{~h} \text { reflux }\end{array}} \rightarrow \frac{\mathrm{Li}}{\text { overnight, } 0^{\circ} \mathrm{C}} \rightarrow \frac{\mathrm{MeOH}}{2 \mathrm{~h}, \mathrm{RT}} \longrightarrow$ Nanomaterial

(b) $\mathrm{MgSi}+\mathrm{Br}_{2} \frac{\mathrm{n} \text {-Octane }}{\begin{array}{l}1.2 \mathrm{~h} \mathrm{RT} \\ 2.18 \mathrm{~h} \text { reflux }\end{array}} \rightarrow \frac{\mathrm{Li}}{\text { overnight, } 0^{\circ} \mathrm{C}} \rightarrow \frac{\mathrm{MeOH}}{2 \mathrm{~h}, \mathrm{RT}} \rightarrow$ Nanomaterial

Scheme 1 Synthesis of Si QDs (a) and the control (b). 


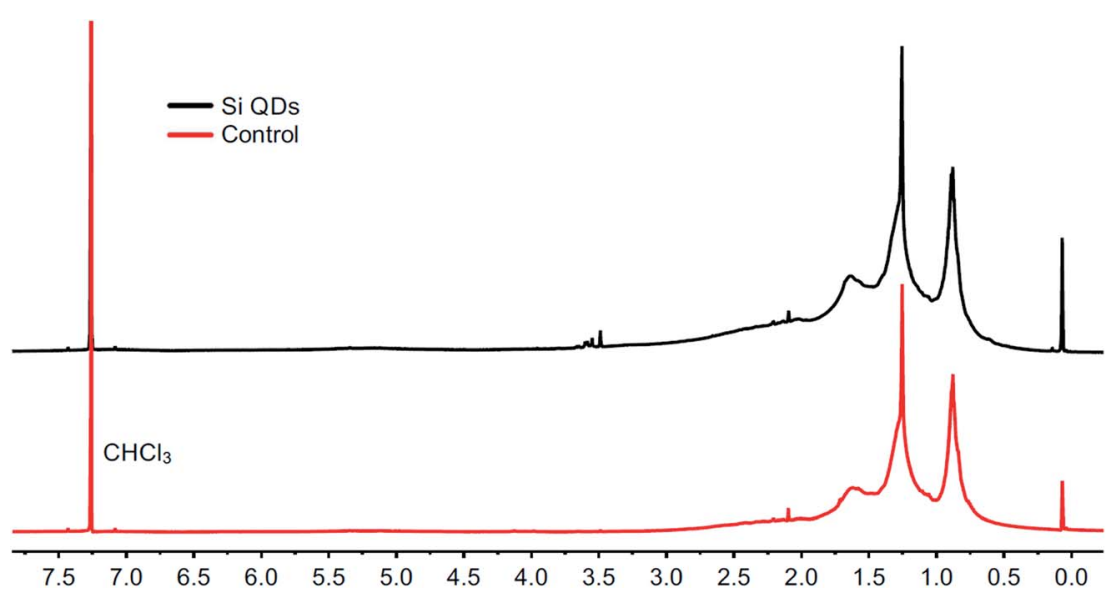

Fig. $1{ }^{1} \mathrm{H}$ NMR spectra from the Si QD synthesis (top) and the control synthesis (bottom) in chloroform-d.

The FTIR spectrum of the Si QDs (see Fig. 2) corresponds well with those from previous literature, although $\mathrm{OH}$ vibrations around $3425 \mathrm{~cm}^{-1}$ were not observed in previous reports, ${ }^{52}$ or only to a minor extent. ${ }^{53}$ Vibrations around $2927 \mathrm{~cm}^{-1}$ correspond to $\mathrm{CH}_{2}$ and $\mathrm{CH}_{3}$ groups, vibrations at $1456 \mathrm{~cm}^{-1}$ to $\mathrm{CH}_{2}$ and $\mathrm{CH}_{3}$ groups, and vibrations at $1377 \mathrm{~cm}^{-1}$ to $\mathrm{CH}_{3}$ groups; all of them are characteristic for alkyl functionalities. Vibrations corresponding to $\mathrm{Si}-\mathrm{CH}_{2}$ bonds have been reported before to appear at approximately $1260 \mathrm{~cm}^{-1}$ and $1460 \mathrm{~cm}^{-1,49,64,89,90}$ but are generally weak. Small vibrations were indeed observed at $1261 \mathrm{~cm}^{-1}$, which might be related to $\mathrm{Si}-\mathrm{CH}_{2}$ vibrations, although it is not strong evidence for $\mathrm{Si}$ QDs, as it may also be assigned to other species. Vibrations at $1705 \mathrm{~cm}^{-1}$ are also present, which has been observed earlier. ${ }^{52,53}$ However, the nature of these species remains unknown, as these vibrations were not assigned. The distinctive vibrations at $1073 \mathrm{~cm}^{-1}$ are attributed to Si-O bonds, as a result of (partial) oxidation of the Si QDs. ${ }^{4,74}$ Notably, this signal was also observed in the control, which makes

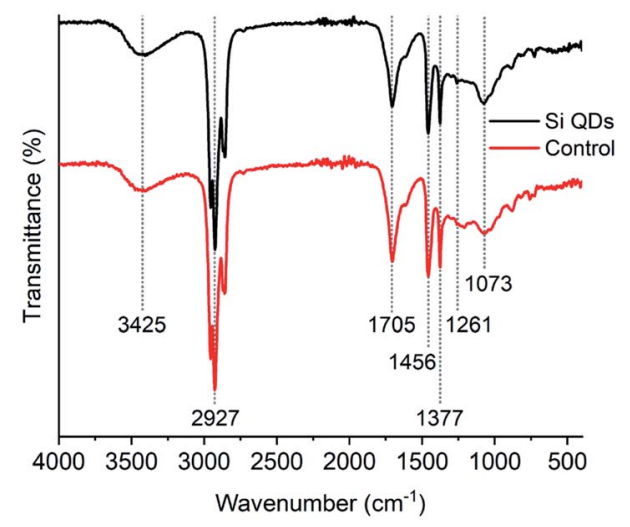

Fig. 2 FTIR spectra of Si QDs and the control. 
the assignment of these vibrations as unique for Si QDs uncertain. Moreover, no discernible differences between the Si QDs and the control were observed.

The samples were further analyzed with TEM, as shown in Fig. 3. It can be observed that non-crystalline nanosized particles are present (size not quantified) in both cases. It should be mentioned that both TEM grids were evaluated until areas were found with high contrast, given that these images do not represent the full sample. However, this was performed in order to show the notable resemblance with Si QDs reported previously. ${ }^{53}$ For the control, it can be excluded that these particles on TEM are Si QDs, indicating that mere particle observations using TEM do not infer the presence of Si QDs by default. Particles might be aggregates of organic matter, carbon dots, or a result of TEM sample preparation. ${ }^{79,80,85}$ Fluorescence by quantum confinement effects needs to be confirmed, regardless of whether the Si QDs are amorphous ${ }^{91}$ or crystalline. ${ }^{92}$

Elemental analysis was performed in order to examine the sample composition, and the presence of silicon in particular, as shown in Table S1. $\dagger$ A Si amount of $2.2 \mathrm{wt} \%$ was found for the Si QDs, while for the control a Si amount of $0.4 \mathrm{wt} \%$ was determined. In both samples, carbon (>60 wt\%) and bromine ( $>3 \mathrm{wt} \%)$ were observed. In combination with the product yield, the reaction efficiency for silicon can be derived. At best, less than $1 \mathrm{mg}$ of elemental Si from the starting material $\left(\mathrm{Mg}_{2} \mathrm{Si}\right)$ can be traced back to the Si QDs, which is equal to $2.2 \%$ conversion of $\mathrm{Mg}_{2} \mathrm{Si}$. Considering the (slightly) higher Si content in Si QDs, conversion of $\mathrm{Mg}_{2} \mathrm{Si}$ may have occurred, but is only marginal.

XPS analysis was performed to obtain more knowledge on the type of $\mathrm{Si}$ bonding in particular, and further confirm the presence of silicon (see Fig. 4 and S1-S4 $\dagger$ ). After deconvolution, a binding energy of $102.2 \mathrm{eV}$ was observed for silicon in the Si QDs, and a binding energy of $102.4 \mathrm{eV}$ was determined for the control. In the case of the control, Si arose from minor impurities, and therefore will be neglected. Binding energies around 102.2 eV were found for the Si QDs, indicating that no fully oxidized $\mathrm{SiO}_{2}$ species were present, as binding energies would be observed around $\sim 103.5 \mathrm{eV} \cdot{ }^{25,56}$ However, XPS data for siloxanes shows similar binding energies to that for Si QDs, ${ }^{93}$ whereas such materials consist primarily of $\mathrm{Si}-\mathrm{O}$ bonds. In both the control and the Si QDs, it is not unlikely that similar oxygen-bound silicon material is present. However, most importantly, binding energies at $\sim 99.5 \mathrm{eV}$ (depending on the oxidation state), corresponding to $\mathrm{Si}-\mathrm{Si}$ bonds, are absent, though expected in the case of Si QDs. ${ }^{25,77}$ Hence, the presence of appreciable amounts of Si QDs cannot be confirmed.
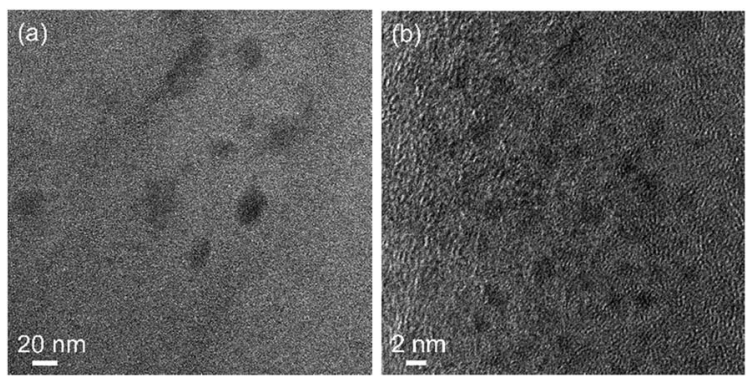

Fig. 3 TEM images of Si QDs (a) and the control (b). 


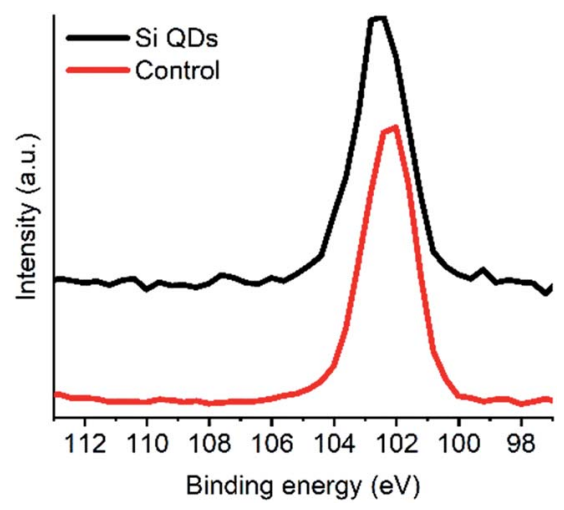

Fig. 4 XPS spectra Si QDs and the control (Si 2p scan).

The absorbance and fluorescence of Si QDs and the control are depicted in Fig. 5. The Si QDs revealed no characteristic features in the absorbance, as described earlier. ${ }^{52,53}$ While it is known that QDs show features that correlate to their size, proposed for Si QDs as well, ${ }^{94}$ the absence of features may also be an effect of a heterogeneous particle size distribution. However, the similarity with the control is of more concern, indicating no discernable differences.

The fluorescence properties of the samples were subsequently assessed, as shown in Fig. 5. Under visible light, both samples are colorless to pale yellow, while under UV illumination bright blue/green fluorescence can be observed, consistent with the emission observed in the excitation plots. For the Si QDs, the fluorescence spectra are comparable to those reported before, ${ }^{52}$ although the emission observed for the Si QDs at lower excitation wavelengths is somewhat stronger. For excitation wavelengths between $\sim 260-320 \mathrm{~nm}$, the emission wavelength is centered around $\sim 380 \mathrm{~nm}$, while for higher excitation wavelengths, the emission was red-shifted and rapidly decreased in intensity. Thus, below excitation wavelengths of $320 \mathrm{~nm}$, excitation-independent emission dominates, while for higher excitation wavelengths, excitation-dependent emission is stronger. The reported emission peak maximum $(383 \mathrm{~nm})$ in the literature is consistent with the maximum in our spectra. However, the most effective excitation wavelength was
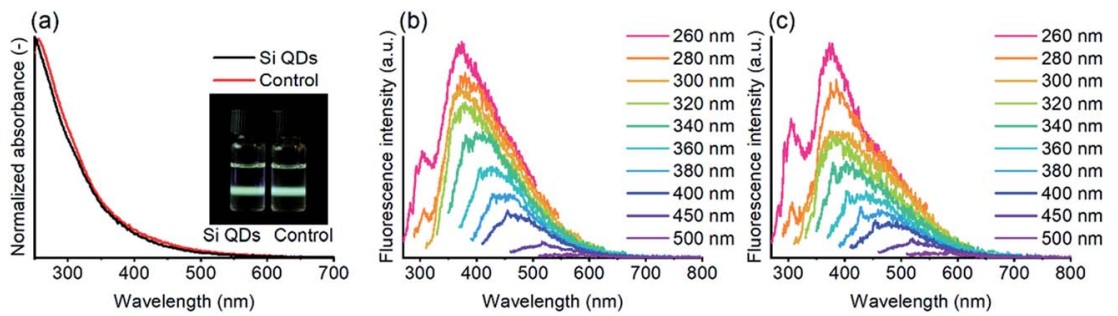

Fig. 5 Absorbance of Si QDs and the control, with an insert showing both samples under UV (excitation $405 \mathrm{~nm}$ ) illumination (a), and excitation plots of Si QDs (b) and the control (c) in ethyl acetate, with the excitation wavelength ranging from 260 to $500 \mathrm{~nm}$ (see also Fig. $55 \dagger$ ). 
reported to be at $\sim 320 \mathrm{~nm}$, whilst in our case, the lowest excitation wavelength at $260 \mathrm{~nm}$ was the most efficient. The most plausible explanation would be that in the current work, more blue-emitting matter was produced due to slight experimental differences. For instance, shorter reaction times or slightly lower temperatures might possibly result in more blue-emitting material..$^{80}$ In Fig. S5, $\dagger$ the strongly overlapping emission of the Si QDs and the control can be observed, regardless of excitation wavelength. The fluorescence emission of the control cannot originate from Si QDs, yet still the spectra from Si QDs show the same identical emission spectra as the control, confirming that fluorescence emission in both the Si QDs and control samples is (mostly) determined by species other than Si QDs.

The quantum yield (QY) of the Si QDs, at an excitation wavelength of $350 \mathrm{~nm}$, was determined via a relative method. ${ }^{95}$ The QY was found to be $2.2 \%$, which is comparable with previously published data. ${ }^{53}$ For the control, the QY was somewhat lower at $1.1 \%$.

By means of size exclusion chromatography (SEC) it was possible to separate the materials based on their size, as shown in Fig. 6, and evaluate the optical properties accordingly. For the Si QDs, it was observed that two populations are present, while only the smaller material showed significant fluorescence. Complete SEC traces of absorbance and fluorescence can be found in Fig. S6. $\uparrow$ For fluorescence emission, it can be observed that for the Si QDs and the control, the fluorescence spectra were very comparable. In addition, both materials showed size-dependent fluorescence, which before was thought to be a result of quantum
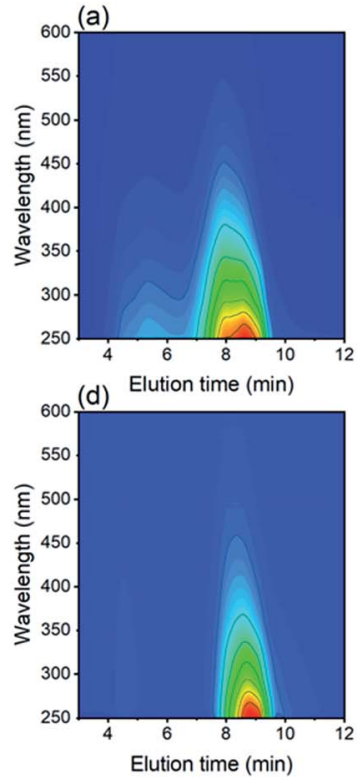

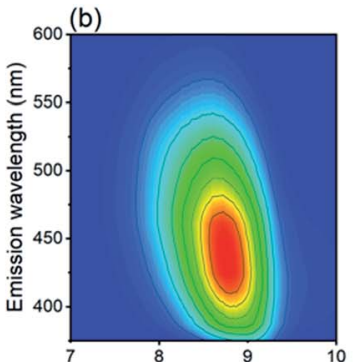

(e) Elution time $(\mathrm{min})$

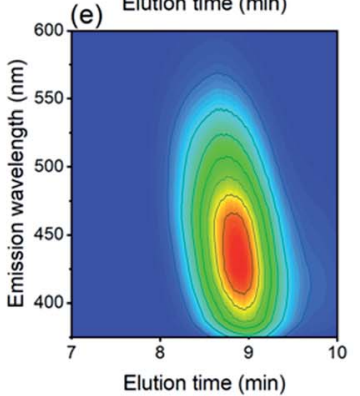

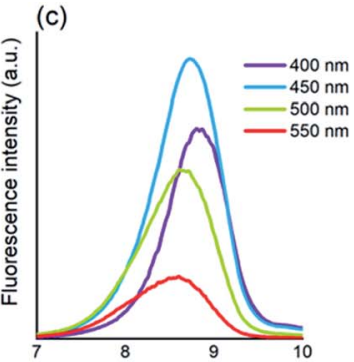

(f)

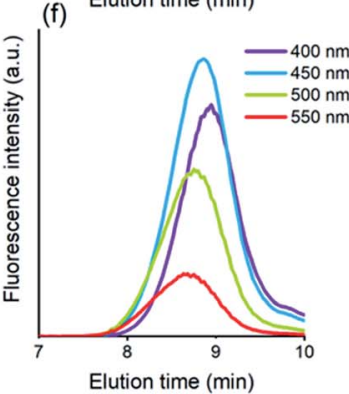

Fig. 6 SEC traces of absorbance (a) and fluorescence ( $b$ and c) of Si QDs, and absorbance (d) and fluorescence (e and f) of the control. The intensity of absorbance and fluorescence increases from blue to red in the contour plots. The emission intensities in (c) and (f) were measured at four different emission wavelengths. 
confinement. ${ }^{52}$ Emission at $400 \mathrm{~nm}$ was strongest at $8.87 \mathrm{~min}$, while emission at $550 \mathrm{~nm}$ was strongest at an elution time of $8.60 \mathrm{~min}$. Therefore, it can be concluded that solely size-dependent emission is not proof of Si QD emission.

There are, however, more notable deviations in the case of the absorbance spectra. It can be observed in Fig. 6 that for both samples, absorbance is observed over a much broader range $(\sim 3.5-10 \mathrm{~min})$ than fluorescence $(\sim 8-9.5 \mathrm{~min})$, indicating the presence of an emissive fraction at longer elution times, and a less- or non-emissive fraction at shorter elution times. The absorbance for Si QDs is much stronger than for the control at short elution times ( 3.5-7 $\mathrm{min})$, although also in the control absorbance was observed to a minor extent (see also Fig. S7 $\dagger$ ). Size fractionation of this material has been carried out, revealing enhanced silicon content ( $0.6 \mathrm{wt} \%$ vs. $0.2 \mathrm{wt} \%),{ }^{96}$ however, this species does not display strong fluorescence. Remarkably, a similar effect was also found in carbon dot synthesis, where size-separation was performed via dialysis. ${ }^{79}$ Smaller materials $(<1 \mathrm{kDa})$ showed strong fluorescence, while the larger materials $(\geq 50 \mathrm{kDa})$ showed much weaker fluorescence. This again suggests that in the samples investigated here, the fluorescence emission is likely caused by small fluorescent materials other than Si QDs.

The findings that the reactions carried out in the presence and absence of $\mathrm{Mg}_{2} \mathrm{Si}$ result in products with almost identical properties raises questions concerning the actual nature of the material formed. Therefore, determining the origin of these by-products was attempted. Direct exposure of $\mathrm{Mg}_{2} \mathrm{Si}$ to $\mathrm{Br}_{2}$ in the absence of a solvent with overnight stirring did not result in any noticeable reaction. Alternatively, elemental (insoluble) silicon may have been formed, as presumed for the Si QDs synthesis carried out in glyme. ${ }^{52}$ In addition, it is known that reactions between $\mathrm{Mg}_{2} \mathrm{Si}$ and aqueous $\mathrm{HCl}$ yield (higher) silanes, ${ }^{\mathbf{9 7}, 98}$ assuming that aqueous $\mathrm{HBr}$ reacts in a similar fashion. Hereunder, it is shown that $\mathrm{HBr}$ is indeed formed during Si QD synthesis, although it remains unclear whether other species (besides octyl bromides and $\mathrm{HBr}$ ) would form and how the reaction might proceed further.

After addition of $\mathrm{Br}_{2}$ to the reaction mixture containing $\mathrm{Mg}_{2} \mathrm{Si}$ and $n$-octane, the color of the $\mathrm{Br}_{2}$ fades. This is also the case in the absence of $\mathrm{Mg}_{2} \mathrm{Si}$. Bromination of alkanes has been demonstrated before, ${ }^{99-102}$ and results in the formation of alkyl bromides and HBr gas. ${ }^{102}{ }^{1} \mathrm{H}$ NMR spectroscopic analysis indeed confirms the formation of octyl bromides, as evidenced by signals at 3.99, 4.04 and $4.13 \mathrm{ppm}$, which were assigned (primarily) to three isomers of secondary octyl bromides (ratios not quantified, see Fig. S8 $\dagger$ ). Formation of considerable amounts of $\mathrm{HBr}$ gas was visually confirmed, as well as through the use of a $\mathrm{pH}$ indicator. Despite considerable evolution of $\mathrm{HBr}$ gas, it should be noted that the solubility of $\mathrm{HBr}$ in $n$-octane is also significant. ${ }^{\mathbf{1 0 3}}$ Formation of octyl bromides was observed before, but was then not considered to be the origin of strongly fluorescent material. ${ }^{53}$ Experimentally, products of the reaction between $n$-octane and $\mathrm{Br}_{2}$ were collected, possible residual $\mathrm{Br}_{2}$ and $\mathrm{HBr}$ were removed by extraction against water, and the organic phase, containing $n$-octane and octyl bromides, was dried over $\mathrm{Na}_{2} \mathrm{SO}_{4}$. Subsequently, this mixture of $n$-octane and octyl bromides was heated to reflux, and fluorescent material was again obtained. This indicates that $\mathrm{HBr}$ and $\mathrm{Br}_{2}$ are likely not essential for formation of fluorescent by-products upon heating, but rather that other, earlier formed species are, e.g. octyl bromides. 
In addition to our critical assessment of Si QD synthesis by means of wetchemical oxidation, we stress that related work on Si QDs should be critically assessed as well, and bottom-up syntheses in particular.

In our view, most essential in Si QD synthesis are two aspects. Firstly, it should be shown convincingly that (crystalline) Si QDs have been synthesized successfully, preferably using an appropriate combination of methods discussed below. ${ }^{26,76}$ Secondly, it should be confirmed that these Si QDs are indeed the true origin of displayed fluorescence. Careful purification and characterization are therefore essential. Frequently, successful fabrication of (size-tunable) fluorescent Si QDs is claimed, almost entirely based on particles observed by TEM in combination with fluorescence spectra. However, as indicated, the presence of (crystalline) particles alone is not direct proof of the formation of fluorescing Si QDs. ${ }^{79,85}$ Furthermore, it is not improbable that side products formed during synthesis might attach to the surface of the true QDs, further complicating observations. ${ }^{80}$

As long as the quantum confinement model is applicable, evidence of a direct relation between determined size and fluorescence emission would be of great value in this regard, for instance achieved by fluorescence spectroscopy on sizeseparated samples. Alternatively, differently sized particles may be prepared and their respective sizes and fluorescence emissions can be assessed., ${ }^{\mathbf{4 , 1 0 4}}$ Unfortunately, surface effects influencing fluorescence, and thus reducing or eliminating the effect of size on emission, especially towards smaller sizes, may complicate such characterization methods. ${ }^{105}$

Veinot and coworkers provided guidelines for the evaluation of Si QDs, ${ }^{26}$ which are in line with a more general approach published on inorganic colloidal materials. ${ }^{106}$ In fact, characterization can be performed almost inexhaustibly, and a combination of techniques will always be required due to the complex nature of Si QDs. ${ }^{26}$ Most conveniently, information regarding crystallinity can be obtained using TEM $^{107,108}$ and XRD, ${ }^{77}$ size is readily assessed by TEM and SAXS, ${ }^{25,66}$ while the nature of Si-bonding can be determined using Raman spectroscopy, ${ }^{109}$ FTIR $^{\mathbf{1 0 8 , 1 1 0}}$ and XPS. ${ }^{25}$ The optical properties can be ascertained through fluorescence and UV-vis spectroscopic analysis, while chemical composition can be determined by elemental analysis and, trivially but importantly, by reporting product yields. Further insights can be gained from techniques such as dynamic light scattering, ${ }^{111}$ atomic force microscopy, ${ }^{112}$ thermogravimetric analysis, ${ }^{113}$ NMR spectroscopy, ${ }^{77}$ fluorescence lifetime experiments, ${ }^{114}$ ultracentrifugation, ${ }^{115}$ sizeselective precipitation, ${ }^{105}$ and size exclusion chromatography. ${ }^{28,116}$

For purification, chromatographic separation based on size (e.g. SEC) or polarity (e.g. HPLC) are powerful tools for removing (fluorescent) by-products, ${ }^{80,117}$ while dialysis may be convenient as well. ${ }^{79}$ It has been shown that purification in the field of nanotechnology has been frequently performed only to a limited extent, if at all, leading to misinterpretations. ${ }^{78}$ For instance, centrifugation or filtration alone is typically insufficient. ${ }^{79}$ Dialysis, especially when using a molecular weight cut-off below $20 \mathrm{kDa}$, is not recommended, due to its limited ability to remove organic residues. ${ }^{79}$

Our studies indicate that the reaction solvents may not be as inert as generally considered, and can induce fluorescence (possibly in combination with other reactants), especially when heat or microwave radiation is applied. For instance, it has been shown that refluxing ethylene glycol readily results in fluorescent material, ${ }^{84}$ as reported for poly(ethylene glycol) as well. ${ }^{118}$ The combination of sodium and 
refluxing diglyme has also been demonstrated to lead to fluorescent by-products (suggested to be polymerized diglyme). ${ }^{119}$ These by-products could also form during a Si QD synthesis where sodium naphthalide is reacted in refluxing glyme. ${ }^{41}$ $\mathrm{Gu}$ et al. obtained fluorescent materials when heating a series of solvents (DMF, DMAc, xylene, $n$-hexane and cyclohexane) to $260{ }^{\circ} \mathrm{C}$ in an autoclave. ${ }^{120}$ Generally, reaction temperatures in wet-chemical Si QD syntheses (e.g. glyme at $85{ }^{\circ} \mathrm{C},{ }^{52,64} n$ octane at $126{ }^{\circ} \mathrm{C},{ }^{52,53} \mathrm{DMF}$ at $153{ }^{\circ} \mathrm{C}$ (ref. 57 and 58)) and microwave-assisted Si QD syntheses (e.g. DMSO, DMF, acetonitrile at $160^{\circ} \mathrm{C},{ }^{33}$ glycerol at $180^{\circ} \mathrm{C}^{121}$ and DMF at $275^{\circ} \mathrm{C}$ (ref. 34)) are slightly lower. However, it is important to rule out the formation and/or insufficient removal of such fluorescent materials when comparable conditions are employed. For example, microwave-assisted synthesis in DMF at $275^{\circ} \mathrm{C}$ was confirmed to result in the formation of fluorescent by-products. ${ }^{34}$ Hence the requirement for effective purification. Straightforward control experiments (without a source of silicon, in the case of Si QDs) would help to confirm that the observed fluorescence does not originate from by-products. ${ }^{76,84}$

\section{Conclusions}

In the assessment of the wet-chemical oxidation of $\mathrm{Mg}_{2} \mathrm{Si}$ with $\mathrm{Br}_{2}$ we observed the formation of fluorescent nanomaterials, even in the absence of a silicon precursor. We demonstrated that the materials formed in the presence and absence of $\mathrm{Mg}_{2} \mathrm{Si}$ display very comparable optical properties. Not only were the optical properties similar, but other physicochemical properties, as determined using other characterization techniques, were found to be similar. In fact, only SEC characterization and elemental analysis revealed minute differences. However, no evidence was found supporting the formation of Si QDs in substantial amounts. No crystallinity corresponding to silicon was observed, no size-dependent emission different from the control without $\mathrm{Mg}_{2} \mathrm{Si}$ was shown, and no proof of Si-Si bonds was observed. We suggest that wet-chemical syntheses, especially those involving heating, can lead to the formation of fluorescent by-products with similar optical properties to those of Si QDs. The presence of fluorescent impurities might not easily be distinguished or detected due to a generally high content of organic material in the samples and there being no need for the carbon material to be crystalline to show size-dependent properties. ${ }^{78}$

The optical properties observed in Si QD synthesis should be convincingly linked to the actual nanomaterial. This may be achieved by appropriate purification by chromatography and a combination of characterization techniques to determine Si QD crystallinity, size, bonds, optical properties, composition and product yields. Considerations regarding Si QDs may also be extended to other types of fluorescent nanoparticles.

\section{Conflicts of interest}

There are no conflicts to declare.

\section{Acknowledgements}

We thank Dr Rico Keim for performing TEM measurements and Mr Gerard Kip for performing XPS measurements. 


\section{References}

1 A. D. Yoffe, Adv. Phys., 2001, 50, 1-208.

2 C. B. Murray, D. J. Norris and M. G. Bawendi, J. Am. Chem. Soc., 1993, 115, 8706-8715.

3 A. Gupta, M. T. Swihart and H. Wiggers, Adv. Funct. Mater., 2009, 19, 696-703.

4 C. M. Hessel, E. J. Henderson and J. G. C. Veinot, Chem. Mater., 2006, 18, 6139-6146.

5 D. Norris and M. Bawendi, Phys. Rev. B: Condens. Matter Mater. Phys., 1996, 53, 16338-16346.

6 Y. Shirasaki, G. J. Supran, M. G. Bawendi and V. Bulović, Nat. Photonics, 2013, 7, 13-23.

7 M. Dasog, G. B. De Los Reyes, L. V. Titova, F. A. Hegmann and J. G. C. Veinot, ACS Nano, 2014, 8, 9636-9648.

8 X. Gao, L. Yang, J. A. Petros, F. F. Marshall, J. W. Simons and S. Nie, Curr. Opin. Biotechnol., 2005, 16, 63-72.

9 Z. Ni, S. Zhou, S. Zhao, W. Peng, D. Yang and X. Pi, Mater. Sci. Eng., R, 2019, 138, 85-117.

10 F. Priolo, T. Gregorkiewicz, M. Galli and T. F. Krauss, Nat. Nanotechnol., 2014, 9, 19-32.

11 P. V. Kamat, J. Phys. Chem. C, 2008, 112, 18737-18753.

12 F. Maier-Flaig, J. Rinck, M. Stephan, T. Bocksrocker, M. Bruns, C. Kübel, A. K. Powell, G. A. Ozin and U. Lemmer, Nano Lett., 2013, 13, 475-480.

13 E. J. Henderson, A. J. Shuhendler, P. Prasad, V. Baumann, F. Maier-Flaig, D. O. Faulkner, U. Lemmer, X. Y. Wu and G. A. Ozin, Small, 2011, 7, 25072516.

14 F. Erogbogbo, K. T. Yong, I. Roy, G. X. Xu, P. N. Prasad and M. T. Swihart, ACS Nano, 2008, 2, 873-878.

15 U. Resch-Genger, M. Grabolle, S. Cavaliere-Jaricot, R. Nitschke and T. Nann, Nat. Methods, 2008, 5, 763-775.

16 X. Cheng, S. B. Lowe, P. J. Reece and J. J. Gooding, Chem. Soc. Rev., 2014, 43, 2680-2700.

17 X. Michalet, F. F. Pinaud, L. A. Bentolila, J. M. Tsay, S. Doose, J. J. Li, G. Sundaresan, A. M. Wu, S. S. Gambhir and S. Weiss, Science, 2005, 307, 538-544.

18 I. L. Medintz, H. T. Uyeda, E. R. Goldman and H. Mattoussi, Nat. Mater., 2005, 4, 435-446.

19 K. D. Wegner and N. Hildebrandt, Chem. Soc. Rev., 2015, 44, 4792-4834.

20 L. M. Kustov, P. V. Mashkin, V. N. Zakharov, N. B. Abramenko, E. Y. Krysanov, L. A. Aslanov and W. Peijnenburg, Environ. Sci.: Nano, 2018, 5, 2945-2951.

21 M. Dasog, J. Kehrle, B. Rieger and J. G. C. Veinot, Angew. Chem., Int. Ed., 2016, 55, 2322-2339.

22 P. Reiss, M. Carrière, C. Lincheneau, L. Vaure and S. Tamang, Chem. Rev., 2016, 116, 10731-10819.

23 A. S. Heintz, M. J. Fink and B. S. Mitchell, Adv. Mater., 2007, 19, 3984-3988.

24 K. Sato, H. Tsuji, K. Hirakuri, N. Fukata and Y. Yamauchi, Chem. Commun., 2009, 3759-3761. 
25 C. M. Hessel, D. Reid, M. G. Panthani, M. R. Rasch, B. W. Goodfellow, J. Wei, H. Fujii, V. Akhavan and B. A. Korgel, Chem. Mater., 2012, 24, 393-401.

26 R. J. Clark, M. Aghajamali, C. M. Gonzalez, L. Hadidi, M. A. Islam, M. Javadi, M. H. Mobarok, T. K. Purkait, C. J. T. Robidillo, R. Sinelnikov, A. N. Thiessen, J. Washington, H. Yu and J. G. C. Veinot, Chem. Mater., 2017, 29, 80-89.

27 X. Li, Y. He, S. S. Talukdar and M. T. Swihart, Langmuir, 2003, 19, 8490-8496. 28 G. Ledoux, J. Gong, F. Huisken, O. Guillois and C. Reynaud, Appl. Phys. Lett., 2002, 80, 4834-4836.

29 L. Mangolini, E. Thimsen and U. Kortshagen, Nano Lett., 2005, 5, 655-659.

30 F. Kunze, S. Kuns, M. Spree, T. Hülser, C. Schulz, H. Wiggers and S. M. Schnurre, Powder Technol., 2019, 342, 880-886.

31 J. D. Holmes, K. J. Ziegler, R. C. Doty, L. E. Pell, K. P. Johnston and B. A. Korgel, J. Am. Chem. Soc., 2001, 123, 3743-3748.

32 Y. Zhong, F. Peng, F. Bao, S. Wang, X. Ji, L. Yang, Y. Su, S. T. Lee and Y. He, J. Am. Chem. Soc., 2013, 135, 8350-8356.

33 S. P. Pujari, H. Driss, F. Bannani, B. Van Lagen and H. Zuilhof, Chem. Mater., 2018, 30, 6503-6512.

34 T. M. Atkins, A. Thibert, D. S. Larsen, S. Dey, N. D. Browning and S. M. Kauzlarich, J. Am. Chem. Soc., 2011, 133, 20664-20667.

35 B. Song and Y. He, Nano Today, 2019, 26, 149-163.

36 B. F. P. McVey, S. Prabakar, J. J. Gooding and R. D. Tilley, ChemPlusChem, 2017, 82, 60-73.

37 J. R. Heath, Science, 1992, 258, 1131-1133.

38 A. Kamyshny, V. Zakharov, M. Zakharov, A. Yatsenko, S. Savilov, L. Aslanov and S. Magdassi, J. Nanopart. Res., 2011, 13, 1971-1978.

39 L. A. Aslanov, V. N. Zakharov, A. V. Pavlikov, S. V. Savilov, V. Y. Timoshenko and A. V. Yatsenko, Russ. J. Coord. Chem., 2013, 39, 427-431.

40 S. Semlali, B. Cormary, M. L. De Marco, J. Majimel, A. Saquet, Y. Coppel, M. Gonidec, P. Rosa and G. L. Drisko, Nanoscale, 2019, 11, 4696-4700.

41 R. K. Baldwin, K. A. Pettigrew, E. Ratai, M. P. Augustine and S. M. Kauzlarich, Chem. Commun., 2002, 17, 1822-1823.

42 Q. Li, T. Y. Luo, M. Zhou, H. Abroshan, J. Huang, H. J. Kim, N. L. Rosi, Z. Shao and R. Jin, ACS Nano, 2016, 10, 8385-8393.

43 R. D. Tilley and K. Yamamoto, Adv. Mater., 2006, 18, 2053-2056.

44 R. D. Tilley, J. H. Warner, K. Yamamoto, I. Matsui and H. Fujimori, Chem. Commun., 2005, 1833-1835.

45 J. Wang, S. Sun, F. Peng, L. Cao and L. Sun, Chem. Commun., 2011, 47, 49414943.

46 X. Cheng, R. Gondosiswanto, S. Ciampi, P. J. Reece and J. J. Gooding, Chem. Commun., 2012, 48, 11874-11876.

47 J. P. Wilcoxon, G. A. Samara and P. N. Provencio, Phys. Rev. B: Condens. Matter Mater. Phys., 1999, 60, 2704-2714.

48 K. Linehan and H. Doyle, Small, 2014, 10, 584-590.

49 M. Rosso-Vasic, E. Spruijt, B. Van Lagen, L. De Cola and H. Zuilhof, Small, 2008, 4, 1835-1841.

50 N. Lin, Y. Han, L. Wang, J. Zhou, J. Zhou, Y. Zhu and Y. Qian, Angew. Chem., Int. Ed., 2015, 54, 3822-3825.

51 A. Shavel, L. Guerrini and R. A. Alvarez-Puebla, Nanoscale, 2017, 9, 8157-8163. 
52 K. A. Pettigrew, Q. Liu, P. P. Power and S. M. Kauzlarich, Chem. Mater., 2003, 15, 4005-4011.

53 L. Ruizendaal, S. P. Pujari, V. Gevaerts, J. M. J. Paulusse and H. Zuilhof, Chem.-Asian J., 2011, 6, 2776-2786.

54 Q. Liu and S. M. Kauzlarich, Mater. Sci. Eng., B, 2002, 96, 72-75.

55 M. Imamura, J. Nakamura, S. Fujimasa, H. Yasuda, H. Kobayashi and Y. Negishi, Eur. Phys. J. D, 2011, 63, 289-292.

56 C. W. Hsu, D. Septiadi, C. H. Lai, P. Chen, P. H. Seeberger and L. De Cola, ChemPlusChem, 2017, 82, 660-667.

57 X. Zhang, D. Neiner, S. Wang, A. Y. Louie and S. M. Kauzlarich, Nanotechnology, 2007, 18, 095601.

58 B. M. Nolan, T. Henneberger, M. Waibel, T. F. Fässler and S. M. Kauzlarich, Inorg. Chem., 2015, 54, 396-401.

59 D. Neiner, H. W. Chiu and S. M. Kauzlarich, J. Am. Chem. Soc., 2006, 128, 11016-11017.

60 B. Cho, S. Baek, H. G. Woo and H. Sohn, J. Nanosci. Nanotechnol., 2014, 14, 5868-5872.

61 D. Rodríguez Sartori, C. R. Lillo, J. J. Romero, M. Laura Dellarciprete, A. Miñán, M. Fernández Lorenzo De Mele and M. C. Gonzalez, Nanotechnology, 2016, 27, 475704.

62 D. Mayeri, B. L. Phillips, M. P. Augustine and S. M. Kauzlarich, Chem. Mater., 2001, 13, 765-770.

63 R. A. Bley and S. M. Kauzlarich, J. Am. Chem. Soc., 1996, 118, 12461-12462.

64 C. S. Yang, R. A. Bley, S. M. Kauzlarich, H. W. H. Lee and G. R. Delgado, J. Am. Chem. Soc., 1999, 121, 5191-5195.

65 B. Ghosh and N. Shirahata, Sci. Technol. Adv. Mater., 2014, 15, 014207.

66 Y. Yu, G. Fan, A. Fermi, R. Mazzaro, V. Morandi, P. Ceroni, D. M. Smilgies and B. A. Korgel, J. Phys. Chem. C, 2017, 121, 23240-23248.

67 K. Dohnalová, A. Fučíková, C. P. Umesh, J. Humpolíčková, J. M. J. Paulusse, J. Valenta, H. Zuilhof, M. Hof and T. Gregorkiewicz, Small, 2012, 8, 31853191.

68 X. Wen, P. Zhang, T. A. Smith, R. J. Anthony, U. R. Kortshagen, P. Yu, Y. Feng, S. Shrestha, G. Coniber and S. Huang, Sci. Rep., 2015, 5, 12469.

69 R. Sinelnikov, M. Dasog, J. Beamish, A. Meldrum and J. G. C. Veinot, ACS Photonics, 2017, 4, 1920-1929.

70 T. Takagahara and K. Takeda, Phys. Rev. B, 1992, 46, 15578-15581.

71 D. C. Hannah, J. Yang, P. Podsiadlo, M. K. Y. Chan, A. Demortière, D. J. Gosztola, V. B. Prakapenka, G. C. Schatz, U. Kortshagen and R. D. Schaller, Nano Lett., 2012, 12, 4200-4205.

72 K. Dohnalová, A. N. Poddubny, A. A. Prokofiev, W. D. De Boer, C. P. Umesh, J. M. Paulusse, H. Zuilhof and T. Gregorkiewicz, Light: Sci. Appl., 2013, 2, e47.

73 M. Dasog, Z. Yang, S. Regli, T. M. Atkins, A. Faramus, M. P. Singh, E. Muthuswamy, S. M. Kauzlarich, R. D. Tilley and J. G. C. Veinot, ACS Nano, 2013, 7, 2676-2685.

74 A. Gupta and H. Wiggers, Nanotechnology, 2011, 22, 055707.

75 J. Fuzell, A. Thibert, T. M. Atkins, M. Dasog, E. Busby, J. G. C. Veinot, S. M. Kauzlarich and D. S. Larsen, J. Phys. Chem. Lett., 2013, 4, 3806-3812.

76 B. V. Oliinyk, D. Korytko, V. Lysenko and S. Alekseev, Chem. Mater., 2019, 31, 7167. 
77 A. N. Thiessen, M. Ha, R. W. Hooper, H. Yu, A. O. Oliynyk, J. G. C. Veinot and

V. K. Michaelis, Chem. Mater., 2019, 31, 678-688.

78 J. B. Essner and G. A. Baker, Environ. Sci.: Nano, 2017, 4, 1216-1263.

79 J. B. Essner, J. A. Kist, L. Polo-Parada and G. A. Baker, Chem. Mater., 2018, 30, 1878-1887.

80 N. C. Verma, A. Yadav and C. K. Nandi, Nat. Commun., 2019, 10, 2391.

81 Y. Song, S. Zhu, S. Zhang, Y. Fu, L. Wang, X. Zhao and B. Yang, J. Mater. Chem. C, 2015, 3, 5976-5984.

82 C. J. Reckmeier, J. Schneider, Y. Xiong, J. Häusler, P. Kasák, W. Schnick and A. L. Rogach, Chem. Mater., 2017, 29, 10352-10361.

83 V. Štengl and J. Henych, Nanoscale, 2013, 5, 3387-3394.

84 L. Jiang and H. Zeng, Nanoscale, 2015, 7, 4580-4583.

85 S. Khan, A. Sharma, S. Ghoshal, S. Jain, M. K. Hazra and C. K. Nandi, Chem. Sci., 2017, 9, 175-180.

86 L. Ruizendaal, Functional Silicon Nanoparticles, Wageningen University, 2011.

87 W. Biesta, B. Van Lagen, V. S. Gevaert, A. T. M. Marcelis, J. M. J. Paulusse,

M. W. F. Nielen and H. Zuilhof, Chem. Mater., 2012, 24, 4311-4318.

88 I. M. D. Höhlein, J. Kehrle, T. K. Purkait, J. G. C. Veinot and B. Rieger, Nanoscale, 2015, 7, 914-918.

89 J. H. Warner, A. Hoshino, K. Yamamoto and R. D. Tilley, Angew. Chem., Int. Ed., 2005, 44, 4550-4554.

90 A. Shiohara, S. Hanada, S. Prabakar, K. Fujioka, T. H. Lim, K. Yamamoto, P. T. Northcote and R. D. Tilley, J. Am. Chem. Soc., 2010, 132, 248-253.

91 N. M. Park, C. J. Choi, T. Y. Seong and S. J. Park, Phys. Rev. Lett., 2001, 86, 1355-1357.

92 W. L. Wilson, P. F. Szajowski and L. E. Brus, Science, 1993, 262, 1242-1244.

93 M. Ouyang, C. Yuan, R. J. Muisener, A. Boulares and J. T. Koberstein, Chem. Mater., 2000, 12, 1591-1596.

94 R. Intartaglia, K. Bagga, F. Brandi, G. Das, A. Genovese, E. Di Fabrizio and A. Diaspro, J. Phys. Chem. C, 2011, 115, 5102-5107.

95 J. R. Lakowicz, Principles of fluorescence spectroscopy, Springer Science \& Business Media, 2013.

96 Reaction conditions differed from the syntheses presented in this article and concern higher $\mathrm{Mg}_{2} \mathrm{Si}$ concentrations.

97 A. Stock and C. Somieski, Ber. Dtsch. Chem. Ges., 1916, 49, 111-157.

98 H. J. Emeléus and A. G. Maddock, J. Chem. Soc., 1946, 1131-1134.

99 Y. Manabe, Y. Kitawaki, M. Nagasaki, K. Fukase, H. Matsubara, Y. Hino, T. Fukuyama and I. Ryu, Chem.-Eur. J., 2014, 20, 12750-12753.

100 M. S. Kharasch, W. Hered and F. R. Mayo, J. Org. Chem., 1941, 6, 818-829.

101 G. Egloff, R. E. Schaad and C. D. Lowry, Chem. Rev., 1931, 8, 1-80.

102 X. Wang, R. F. Liu, J. Cork, Y. Gu, D. C. Upham, B. Laycock and E. W. McFarland, Ind. Eng. Chem. Res., 2017, 56, 9411-9418.

103 E. R. Boedeker and C. C. Lynch, J. Am. Chem. Soc., 1950, 72, 3234-3236.

104 C. M. Hessel, E. J. Henderson and J. G. C. Veinot, J. Phys. Chem. C, 2007, 111, 6956-6961.

105 M. L. Mastronardi, F. Maier-Flaig, D. Faulkner, E. J. Henderson, C. Kübel, U. Lemmer and G. A. Ozin, Nano Lett., 2012, 12, 337-342.

106 C. J. Murphy and J. M. Buriak, Chem. Mater., 2015, 27, 4911-4913. 
107 M. G. Panthani, C. M. Hessel, D. Reid, G. Casillas, M. José-Yacamán and B. A. Korgel, J. Phys. Chem. C, 2012, 116, 22463-22468.

108 Y. Yu, C. M. Hessel, T. D. Bogart, M. G. Panthani, M. R. Rasch and B. A. Korgel, Langmuir, 2013, 29, 1533-1540.

109 C. M. Hessel, J. Wei, D. Reid, H. Fujii, M. C. Downer and B. A. Korgel, J. Phys. Chem. Lett., 2012, 3, 1089-1093.

110 L. Mangolini and U. Kortshagen, Adv. Mater., 2007, 19, 2513-2519.

111 K. Kůsová, O. Cibulka, K. Dohnalová, I. Pelant, J. Valenta, A. Fǔcíková, K. Žídek, J. Lang, J. Englich, P. Matějka, P. Štěpánek and S. Bakardjieva, ACS Nano, 2010, 4, 4495-4504.

112 M. Rosso-Vasic, E. Spruijt, Z. Popović, K. Overgaag, B. Van Lagen, B. Grandidier, D. Vanmaekelbergh, D. Domínguez-Gutiérrez, L. De Cola and H. Zuilhof, J. Mater. Chem., 2009, 19, 5926-5933.

113 F. Hua, M. T. Swihart and E. Ruckenstein, Langmuir, 2005, 21, 6054-6062.

114 M. Jakob, A. Aissiou, W. Morrish, F. Marsiglio, M. Islam, A. Kartouzian and A. Meldrum, Nanoscale Res. Lett., 2018, 13, 338.

115 J. B. Miller, A. R. Van Sickle, R. J. Anthony, D. M. Kroll, U. R. Kortshagen and E. K. Hobbie, ACS Nano, 2012, 6, 7389-7396.

116 L. Pitkänen and A. M. Striegel, TrAC, Trends Anal. Chem., 2016, 80, 311-320.

117 Y. Shen, M. Y. Gee, R. Tan, P. J. Pellechia and A. B. Greytak, Chem. Mater., 2013, 25, 2838-2848.

118 M. Chen, W. Wang and X. Wu, J. Mater. Chem. B, 2014, 2, 3937-3945.

119 D. E. Harwell, J. C. Croney, W. Qin, J. T. Thornton, J. H. Day, E. K. Hajime and D. M. Jameson, Chem. Lett., 2003, 32, 1194-1195.

120 J. Gu, X. Li, D. Hu, Y. Liu, G. Zhang, X. Jia, W. Huang and K. Xi, RSC Adv., 2018, 8, 12556-12561.

121 H. L. Ye, S. J. Cai, S. Li, X. W. He, W. Y. Li, Y. H. Li and Y. K. Zhang, Anal. Chem., 2016, 88, 11631-11638. 